\title{
High-Contrast Spectroscopy Testbed for Segmented Telescopes
}

\author{
J.R. Delorme ${ }^{a}$, D. Mawet ${ }^{a, b}$, J. Fucik $^{a}$, J.K. Wallace ${ }^{b}$, G. Ruane ${ }^{a}$, N. Jovanovic ${ }^{a}$, \\ N.S. Klimovich ${ }^{a}$, J.D. Llop Sayson ${ }^{a}$, R. Riddle ${ }^{a}$, R. Dekany ${ }^{a}$, D. Encheverri ${ }^{a}$, G. Vasisht ${ }^{b}$, \\ B. Mennesson ${ }^{b}$ \\ ${ }^{a}$ Department of Astronomy, California Institute of Technology, 1200 E. California Blvd., \\ Pasadena, CA, USA 91125; \\ ${ }^{b}$ Jet Propulsion Laboratory, California Institute of Technology, 4800 Oak Grove Drive, \\ Pasadena, CA, USA 91109;
}

\begin{abstract}
The High Contrast Spectroscopy Testbed for Segmented Telescopes (HCST) at Caltech is aimed at filling gaps in technology for future exoplanet imagers and providing the U.S. community with an academic facility to test components and techniques for high contrast imaging with future segmented ground-based (TMT, E-ELT) and space-based telescopes (HabEx, LUVOIR). The HCST will be able to simulate segmented telescope geometries with up to 1021 hexagonal segments and time-varying external wavefront disturbances. It also contains a wavefront corrector module based on two deformable mirrors followed by a classical 3-plane single-stage coronagraph (entrance apodizer, focal-plane mask, Lyot stop) and a science instrument. The back-end instrument will consist of an imaging detector and a high-resolution spectrograph, which is a unique feature of the HCST. The spectrograph instrument will utilize spectral information to characterize simulated planets at the photon-noise limit, measure the chromaticity of new optimized coronagraph and wavefront control concepts, and test the overall scientific functions of high-resolution spectrographs on future segmented telescopes.
\end{abstract}

Keywords: Instrumentation, Testbed, Segmented Telescopes Simulator, High contrast imaging, Wavefront control, High Spectral Resolution, High Dispersion Coronagraphy

\section{INTRODUCTION}

The High Contrast Spectroscopy Testbed for Segmented Telescopes (HCST) has been designed to test high contrast imaging components and techniques in preparation for future segmented ground-based telescope, such as the Thirty Meter Telescope (TMT) and the European Extremely Large Telescope (E-ELT), as well as space-based telescopes including the Habitable Exoplanet Imaging Mission (HabEx $)^{1}$ and the Large UV/Optical/IR Surveyor $(\mathrm{LUVOIR})^{2}$ concepts. The HSCT is designed to replicate the realistic aperture geometries, observing conditions, adaptive optics systems, coronagraphs, and science instruments. The priority is to demonstrate starlight suppression technologies for segmented telescopes ${ }^{3}$ and high dispersion coronagraphy (HDC) techniques. ${ }^{4,5}$

Previously this year, the layout of the HCST was been described in detail in the conference proceeding Delorme \& al. 2017. ${ }^{6}$ In this proceeding, we briefly remind the reader of the design of the HCST in section 2. We then describe the latest design updates in sections 3 and 4. Finally, in section 5 we present the progress made with respect to the alignment of the bench.

\section{HCST LAYOUT}

The HCST is shown in Fig 1 and is divided in several modules: the star/planet simulator (in orange), the telescope simulator (in green), the extreme adaptive optic (in blue), the coronagraphic module (in red) and the science instrument (in black).

J.R. Delorme - E-mail: jdelorme@caltech.edu - Telephone: (1) 6263951571 


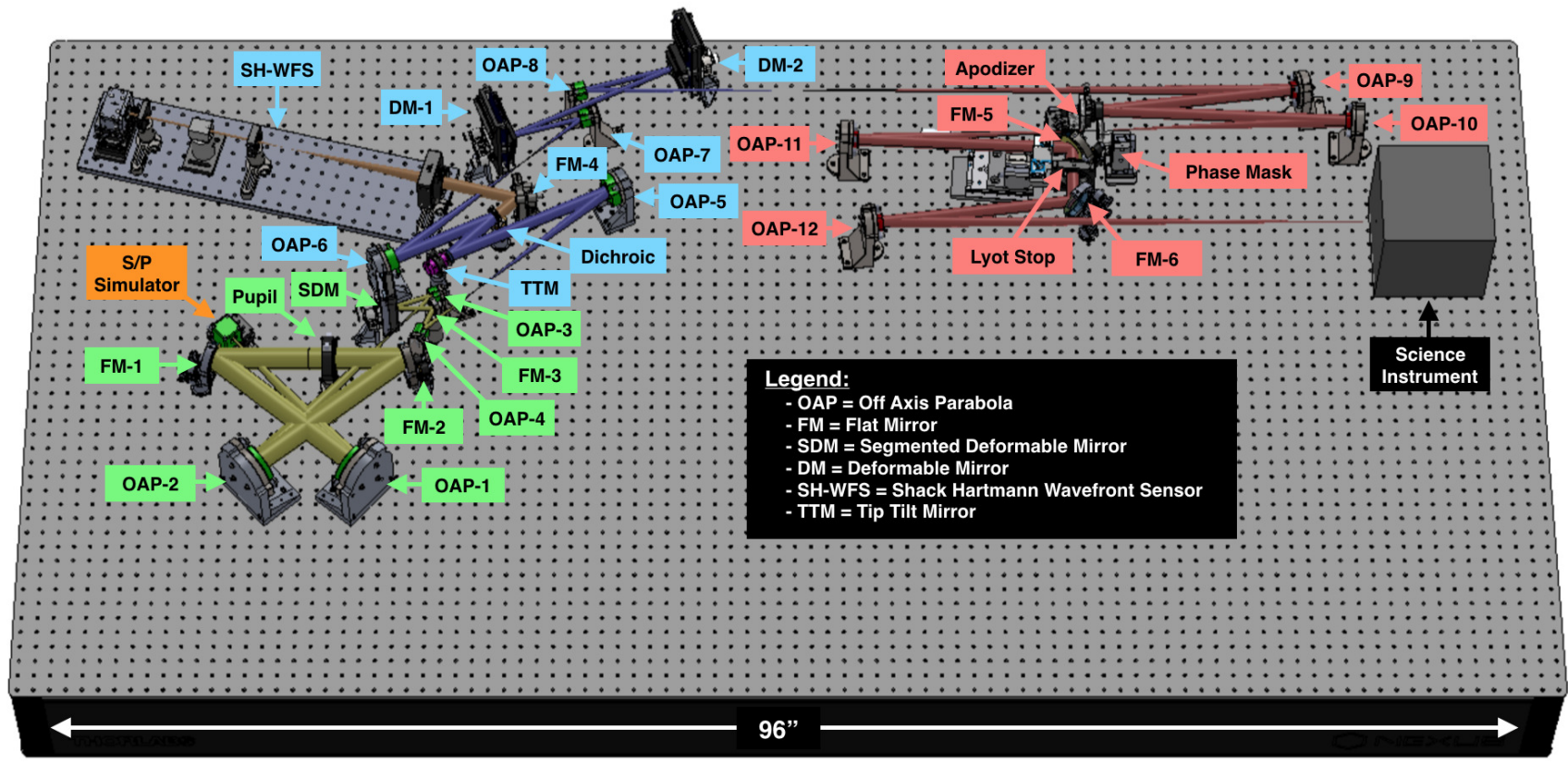

Figure 1. 3D model of the HCST. It is divided in five modules: the Star/Planet simulator (in orange), the telescope simulator (in green) and the extreme adaptive optic (in blue), the coronagraphic module (in red) and the science instrument (in black).

The purpose of the star-planet simulator located at the beginning of the testbed is to generate a star and a planet with tunable angular separation, magnitudes, and spectra. Downstream, the telescope simulator based on: a turbulence simulator (not shown in Fig. 1), a pupil mask described in section 3 and a segmented deformable mirror (SDM), will be used to reproduce most of the actual and future ground-based and space-based segmented telescopes up to 1021 hexagonal segments *. Then an extreme adaptive optic module based on a tip-tilt mirror (TTM), a Shack-Hartmann wavefront sensor (SH-WFS, described in section 4) and two deformable mirrors (DMs) will be used to correct both the phase and amplitude aberrations upstream of the coronagraphic module and the science instrument. These first three modules compose the HCST facility simulator. Several high-contrast imaging components and techniques will be tested in the focal plane, in preparation for future segmented groundbased as well as space-based telescopes. As describd in Delorme \& al 2017, ${ }^{6}$ we are already scheduled to test several concepts of an apodized vortex coronagraph for segmented telescopes ${ }^{7}$ and a fiber injection unit similar to the one currently being designed for the Keck-II telescope. ${ }^{8}$

\section{PUPIL MASK}

The pupil mask located in the first collimated beam of the telescope simulator (between FM-1 and FM-2) will be used to define the main features of the telescope pupil which includes the outer pupil edge, the spiders and the central obstruction. The segments and the gaps between them will be defined by the segmented deformable mirror located in the second collimated beam (between OAP-3 and OAP-4). The pupil mask will be replaced each time we test a new telescope configuration with the HCST (with different spider positions and central obstruction size). Besides the fact that some masks require very small spiders, these masks also need to be freestanding and their edge quality needs to be as good as possible. Therefore, we investigated several techniques to produce these masks. As a first step, we designed a pupil mask to reproduce the main features of the Keck pupil. Indeed, this mask is representative of the masks we want to produce in the future. In order to faithfully reproduce the Keck pupil, the thickness of the spiders was supposed to be $80 \mu \mathrm{m}$. Aware of the difficulty of

${ }^{*}$ The configuration of the HCST shown in Fig. 1 can be used with a 37 and a 163 SDM as well. To use the 1021 SDM, which is much bigger, we need to use another design of the HCST based on the same optics and components. 
producing masks with such small feature sizes, we prepared several designs with several spiders thickness up to $500 \mu \mathrm{m}$. We manufactured these masks using several techniques which include: laser cutting, 3D-printing and electrical discharge machining (EDM). Using 3D-printing techniques, we were not able to produce masks with spider thickness lower than $750 \mu \mathrm{m}$. On top of that, the edge quality of the masks produced was poor and visible by eye. Using laser cutting techniques, we were able to produce masks with fine spiders $(\approx 250 \mu \mathrm{m}$ - see Fig. 2 left). However the edge quality of the spiders was poor. Finally, using the EDM technique we were able to produce a mask with relatively fine spiders $(500 \mu \mathrm{m}$ - see Fig. 2 middle). We also tried to produce a mask with finer spiders $(200 \mu \mathrm{m}$ - see Fig. 2 right). We failed to produce this last mask. However, because we failed during the cutting of the last petal, we are confident that we will be able to produce masks with such small features if required. The next step is to determine the edge quality of the masks produced using the EDM technique and anodize them ${ }^{\dagger}$.

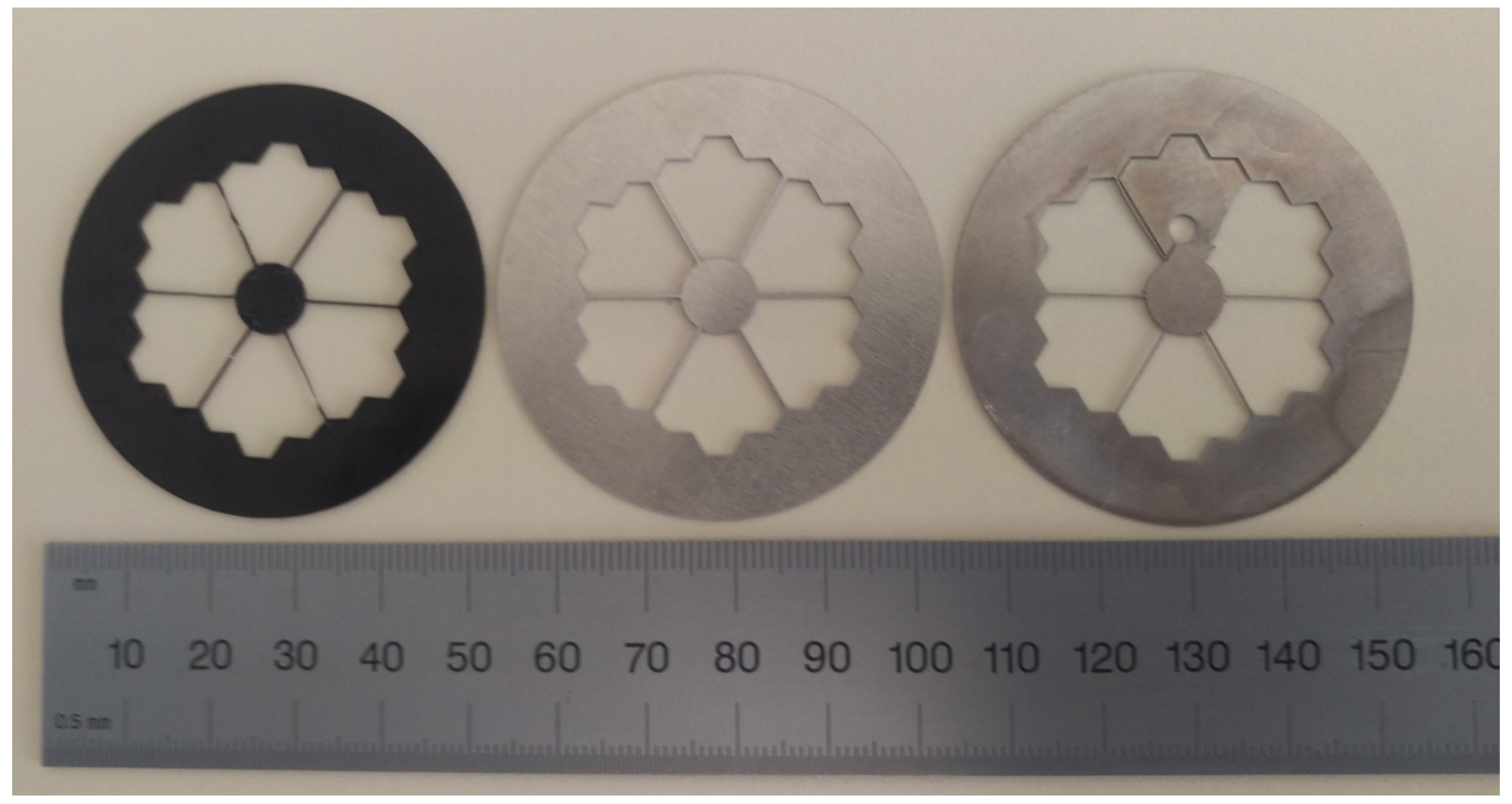

Figure 2. Left: mask produced using laser cutting technique (spider thickness $\approx 250 \mu \mathrm{m}$ ) - Middle: mask produced using EDM (spider thickness $500 \mu \mathrm{m}$ ) - Right: mask produced using EDM (spider thickness $200 \mu \mathrm{m}$ ).

\section{SHACK-HARTMANN WAVEFRONT SENSOR}

The Shack-Hartmann wavefront sensor (see Fig. 3) is a part of the extreme adaptive optic module. In this module, a dichroic located after a tip-tilt mirror (TTM) transmits the visible light to the SH-WFS. The light reflected by the beam splitter is directed towards the two deformable mirrors and the science instrument. The aim of the SH-WFS is to estimate the phase aberrations and correct them using the first deformable mirror (DM-1) and the tip-tilt mirror. Based on a micro-lens array manufactured by Wavefront Science ( $252 \mu \mathrm{m}$ pitch) and a detector manufactured by Teledyne (pixel size: $4.8 \mu \mathrm{m}$ ), several optics are required to adjust the size of the beam. In the HCST configuration presented in this proceeding, the diameter of the input beam is equal to $20.9 \mathrm{~mm}$. Two achromatic lenses (L-1 and L-2) will be used to compress the beam by a factor 5 to illuminate 16 micro-lens apertures across the pupil diameter. Then, a field lens (FL) and a relay lens (RL) will be used to adjust the distance between the spots produced by the micro-lens array. In the focal plane, the distance between the spots needs to be equal to $48 \mu \mathrm{m}$. Indeed, by binning the pixels into groups of 5 -by- 5 , we can increase the

\footnotetext{
${ }^{\dagger}$ Previously, we characterized some test samples, which showed that the edge roughness of a mask produced using the EDM technique can be of the order of $10 \mu \mathrm{m}$ RMS.
} 
detectors frame rate and get 4 binned pixels per spot for wavefront sensing. The data obtained with the detector will be reduced using a software similar to the one written for the Robo-AO project. ${ }^{9,10}$

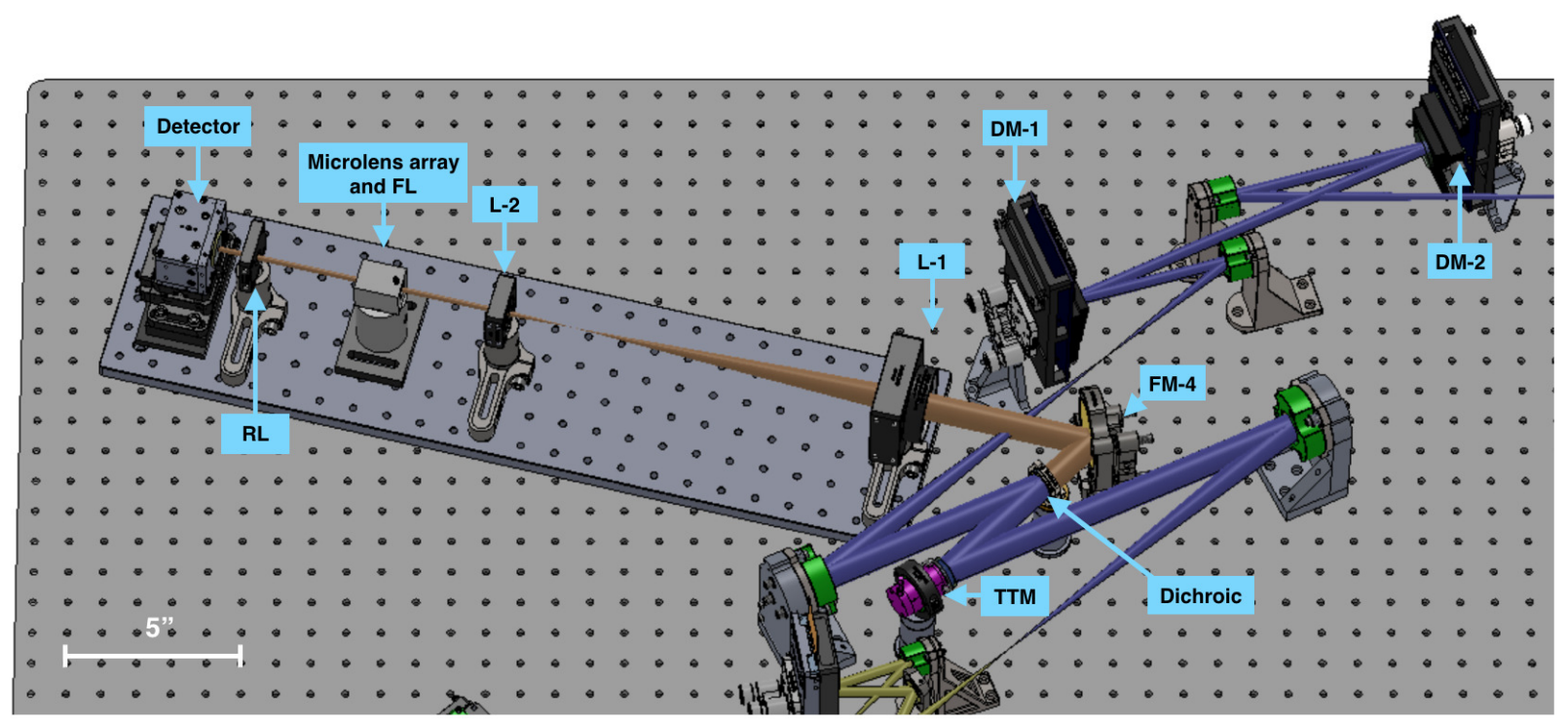

Figure 3. 3D-model of the preliminary design of the Shack-Hartmann wavefront sensor.

\section{INTEGRATION OF HCST}

The HCST is currently undergoing initial integration. To align the first off-axis parabola (OAP-1) and the first flat mirror (FM-1) of the testbed we set up a temporary layout in order to image the point spread function to assess the aberrations. This temporary layout is presented by Fig. 4.

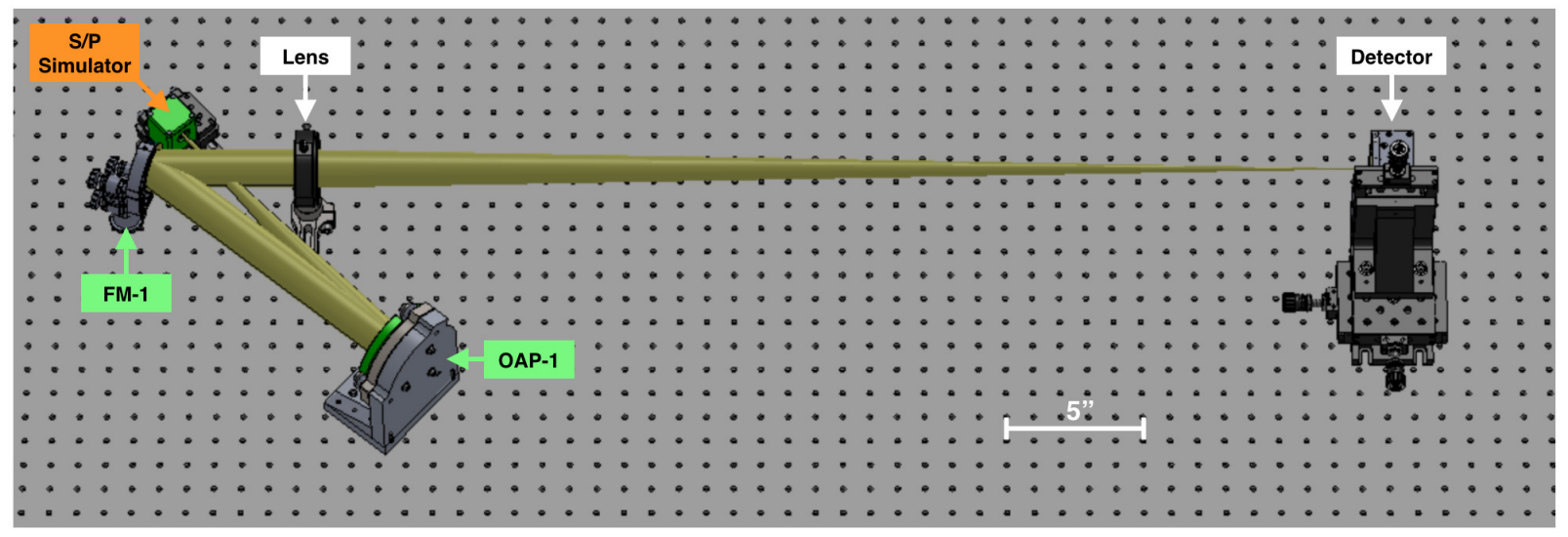

Figure 4. 3D-model of the temporary layout used to aligned OAP-1 and FM-1.

Once the light from the star planet was simulator collimated by OAP-1, the beam was reflected by FM-1. Then a long focal length lens (focal $=1 \mathrm{~m}$ ) focused the light onto a detector. An iris not shown in Fig. 4 was used between the star planet simulator and OAP-1 to constrain the beam. Once aligned we obtained an image using a laser source $(\lambda=635 \mathrm{~nm})$ presented in Fig. 5. This image is presented with a logarithmic scale and was constructed by combining several images obtained for different exposures times.

In this image we can see a structure on the second Airy ring. According to the numerical simulation this structure can be produced by a trefoil phase aberration of a few nanometers RMS. After concluding that this 


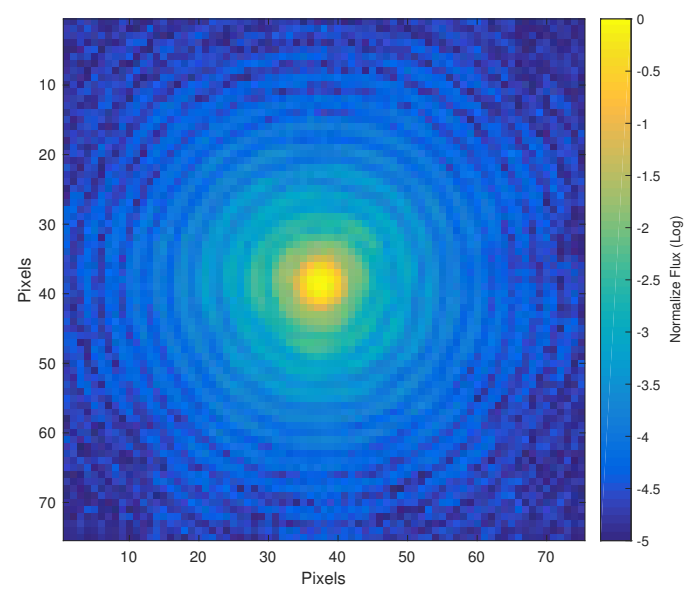

Figure 5. Image obtained after OAP-1 and FM-1.

aberration was not produced by the lens or the detector, we assume that this trefoil was produced by the mount of the flat mirror or the one of the off-axis parabola. Indeed both of the mounts maintained the optics with three point contact. The first design of the mount maintained the OAP in place by using three flexures. In order to dispel any doubts, we characterized the two optics using a Zygo interferometer, as mounted ${ }^{\ddagger}$. These measurements enabled us to determine that the mounts were applying stress to the optics and inducing aberrations. In the case of the flat mirror, it was possible to tune and minimize the stress due to the mount by adjusting the tension in the flexure. However, it was not possible to adjust the tension applied by the springs of the OAP mount. We therefore decided to design a new mount. This new design is based on two hard points and two tunable flexures.

Once the mount was manufactured, we transferred OAP-1 from the initial mount to the new design. Then we aligned the OAP in its new mount in front of the Zygo interferometer and tuned the tension applied by the flexures to minimize the stress on the optic. Finally, we realigned OAP-1 and the flat mirror 1 using the temporary layout presented by Fig. 4. The image obtained after the alignment is presented in Fig. 6 .

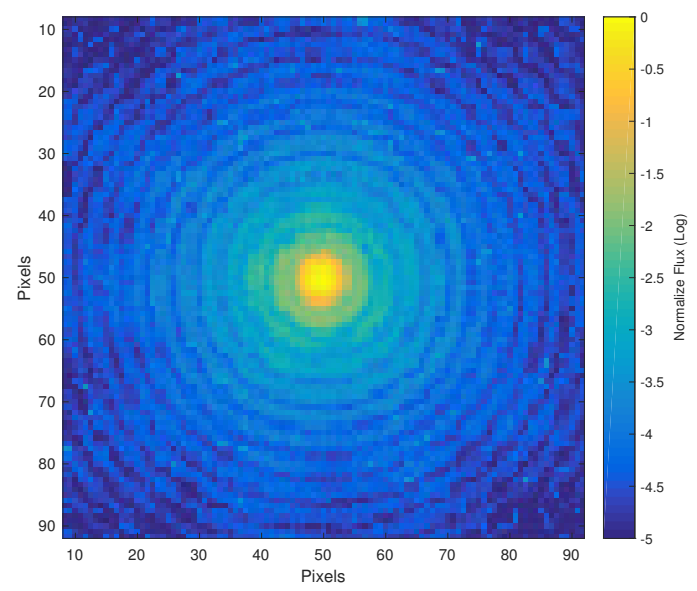

Figure 6. Image obtained after OAP-1 in its new mount and FM-1.

\footnotetext{
${ }^{\ddagger}$ The company who manufactured the optics provided us measurement of the flatness of the optics. Thus, the shapes of the optics were known.
} 
Even if this image, is similar to the one obtained before we modified the OAP mount to be able to adjust the tension applied by the mounts on both optics (OAP-1 and FM-1), we now better understand the behavior and the limit of each component (mounts and optics). The alignment of these first two optics convinced us to modify our alignment protocol. Indeed, all other optics of the HSCT will be characterized as mounted with the zygo before they are aligned within the testbed. The data obtained during the characterization will feed an end-to-end numerical simulation of the bench based on PROPER, ${ }^{11}$ which aims to reproduce HCST as accurately as possible.

\section{CONCLUSION}

Still in its phase of integration, the High Contrast Spectroscopy Testbed for Segmented Telescopes will be operational before the end of the year. Once aligned, the HCST will be able to simulate ground-based and space based segmented telescopes with up to 1021 hexagonal segments. During the first few months following the integration phase, we plan to test apodized vortex coronagraphs for segmented telescopes in order to investigate if this coronagraph can meet the high contrast imaging demands of future segmented space telescopes. We will then test and characterize most of the Keck Planet Imager and Characterizer modules before sending them to the Keck II telescope. Finally after this phase, the HCST will be open to collaborations with the aim of preparing high contrast imaging instruments and techniques dedicated to current and future segmented telescopes.

\section{ACKNOWLEDGMENTS}

The authors would like to acknowledge the financial support of the Heising-Simons foundation. This work was also supported by the Exoplanet Exploration Program (ExEP), Jet Propulsion Laboratory, California Institute of Technology, under contract to NASA.

\section{REFERENCES}

1. B. Mennesson, S. Gaudi, S. Seager, K. Cahoy, S. Domagal-Goldman, L. Feinberg, O. Guyon, J. Kasdin, C. Marois, D. Mawet, M. Tamura, D. Mouillet, T. Prusti, A. Quirrenbach, T. Robinson, L. Rogers, P. Scowen, R. Somerville, K. Stapelfeldt, D. Stern, M. Still, M. Turnbull, J. Booth, A. Kiessling, G. Kuan, and K. Warfield, "The Habitable Exoplanet (HabEx) Imaging Mission: preliminary science drivers and technical requirements," 9904, p. 99040L, 2016.

2. M. R. Bolcar, L. Feinberg, K. France, B. J. Rauscher, D. Redding, and D. Schiminovich, "Initial technology assessment for the Large-Aperture UV-Optical-Infrared (LUVOIR) mission concept study," 9904, p. 99040J, 2016.

3. G. Ruane, J. Jewell, D. Mawet, L. Pueyo, and S. Shaklan, "Apodized vortex coronagraph designs for segmented aperture telescopes," 9912, p. 99122L, 2016.

4. J. Wang, D. Mawet, G. Ruane, R. Hu, and B. Benneke, "Observing Exoplanets with High Dispersion Coronagraphy. I. The Scientific Potential of Current and Next-generation Large Ground and Space Telescopes," Astron. J. 153(4), p. 183, 2017.

5. D. Mawet, J. R. Delorme, G. Ruane, W. Xuan, D. Echeverri, N. Klimovich, M. Randolph, J. Fucik, W. J. K., J. Wang, G. Vasisht, R. Dekany, B. Mennesson, E. Choquet, and E. Serabyn, "Observing Exoplanets with High-dispersion Coronagraphy. II. Demonstration of an Active Single-mode Fiber Injection Unit," Astrophys. J. 838(2), p. 92, 2017.

6. J. R. Delorme, D. Mawet, J. Fucik, N. Jovanovic, J. K. Wallace, R. D. Bartos, P. L. Wizinowich, M. Fitzgerald, S. Lilley, G. Ruane, J. Wang, N. Klimovich, and Y. Xin, "A fiber injection unit for the keck planet imager and characterizer (kpic)," 2017.

7. G. Ruane, D. Mawet, J. Jewell, and S. Shaklan, "Performance and sensitivity of vortex coronagraphs on segmented space telescopes," , Aug. 2017.

8. D. Mawet, J. R. Delorme, N. Jovanovic, J. K. Wallace, R. D. Bartos, P. L. Wizinowich, M. Fitzgerald, S. Lilley, G. Ruane, J. Wang, N. Klimovich, and Y. Xin, "A fiber injection unit for the keck planet imager and characterizer (kpic)," 2017. 
9. C. Baranec, R. Riddle, N. M. Law, A. N. Ramaprakash, S. P. Tendulkar, K. Bui, M. P. Burse, P. Chordia, H. K. Das, J. T. C. Davis, R. G. Dekany, M. M. Kasliwal, S. R. Kulkarni, T. D. Morton, E. O. Ofek, and S. Punnadi, "Bringing the Visible Universe into Focus with Robo-AO," Journal of Vibration Engineering 72, Feb. 2013.

10. C. Baranec, R. Riddle, N. M. Law, A. N. Ramaprakash, S. Tendulkar, K. Hogstrom, K. Bui, M. Burse, P. Chordia, H. Das, R. Dekany, S. Kulkarni, and S. Punnadi, "High-efficiency Autonomous Laser Adaptive Optics," 790, p. L8, July 2014.

11. J. E. Krist, "PROPER: an optical propagation library for IDL," in Optical Modeling and Performance Predictions III, 6675, p. 66750P, Sept. 2007. 